\title{
Endoscopic Injection Sclerotherapy for Rectal Varices with Portal Hypertension
}

\section{Takahiro Sato}

Department of Gastroenterology, Sapporo Kosei General Hospital, Kita 3 Higashi 8, Chuo-ku, Sapporo 060-0033, Japan

\section{Introduction}

Rectal varices represent portal systemic collaterals that are manifested as discrete dilated submucosal veins and constitute a pathway for portal venous flow between the superior rectal veins of the inferior mesenteric system and the middle inferior rectal veins of the iliac system. Although endoscopic injection sclerotherapy (EIS) and endoscopic band ligation (EBL) for esophageal varices are well-established therapies, there is no standard treatment for rectal varices. The aim of this commentary is to evaluate the efficacy of EIS in treating rectal varices.

Data from 102 consecutive patients who underwent treatments for rectal varices were analyzed the clinical outcomes, including complications related to treatments in the Department of Gastroenterology at Sapporo Kosei General Hospital from April 1996 to January 2017. There were 45 males and 57 females, ranging in age from 38 to 91 years (mean, 69.5 year). Fifty-seven of the 102 patients had histories of rectal bleeding, and colonoscopy revealed the high risk sign of variceal rupture in the other 45 patients. EIS was performed weekly using $5 \%$ ethanolamine oleate with iopamidol (5\% EOI) for 90 patients of 102 (Figure 1 and Figure 2). On the other hand, and EBL was done in 10 and percutaneous transhepatic obstruction (PTO) in 2. In all 90 patients, EIS was performed weekly 1 to 5 times (mean, 2.3), and the total amount of sclerosant ranged from 2.0 to $12.0 \mathrm{ml}$ (mean, $5.0 \mathrm{ml}$ ). After EIS, colonoscopy revealed shrinkage of the rectal varices in 89/90 (98.9\%) patients. There were no severe complications such as portal thrombosis and peritonitis. Colonoscopy revealed oozing bleeding from ulcers after EIS in 3 cases, but, no additional treatments were not required. The recurrence rate with bleeding was 2 of the 90 patients (2.2\%), receiving EIS, and 4 of the 10 with EBL (40.0\%), and 1 of the $2(50.0 \%)$ with PTO, respectively.

In conclusion, various medical treatments have been used to control bleeding from rectal varices, but none of these is currently considered to be a standard method. EBL may be suitable as an initial treatment for rectal varices, but it appears that the varices can easily recur

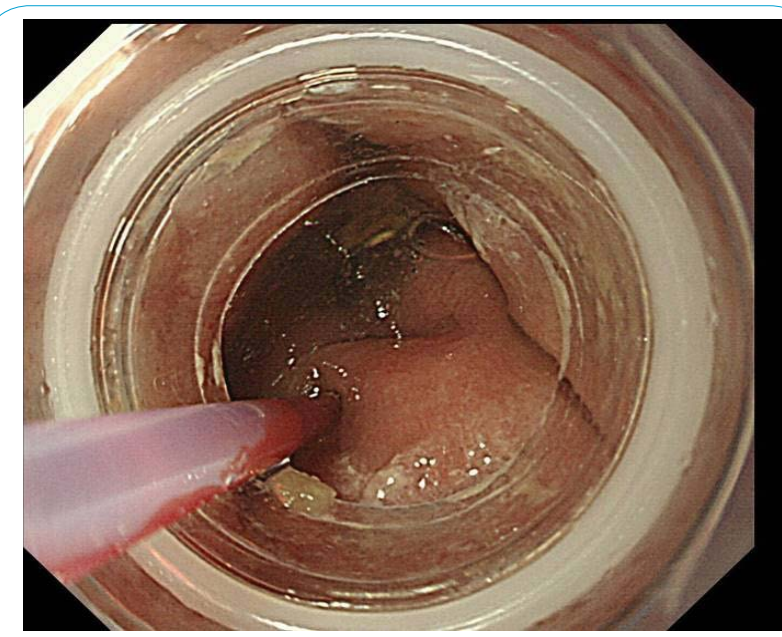

Figure 1: Endoscopic injection sclerotherapy for rectal varices.
Publication History:

Received: April 06, 2017

Accepted: December 02, 2017

Published: December 04, 2017

\section{Keywords:}

Endoscopic injection, Gastroenterology, Colonoscopy, Cisplatin, Thrombosis, Peritonitis

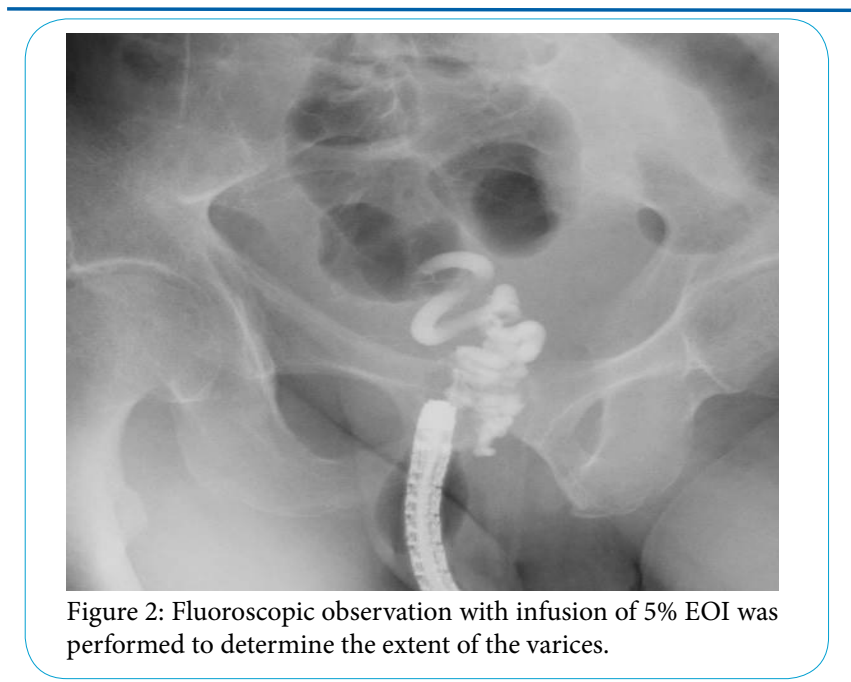

after EBL $[1,2]$. EIS is useful and safety treatment for rectal varices with regard to effectiveness and complications. More investigations are necessary in larger numbers of patients before evidence-based treatment recommendations can be made.

\section{Competing Interests}

The authors have declared that no competing interest exists.

\section{References}

1. Shudo R, Yazaki Y, Sakurai S, Uenishi H, Yamada H, et al. (2000) Endoscopic variceal ligation of bleeding rectal varices: a case report. Dig. Endosc 12: 366-368.

2. Sato T, Yamazaki K, Toyota J, Karino Y, Ohmura T, et al. (2006) The value of the endoscopic therapies in the treatment of rectal varices: a retrospective comparison between injection sclerotherapy and band ligation. Hepatol Res 34: 250-55

"Corresponding Author: Dr. Takahiro Sato, Department of Gastroenterology, Sapporo Kosei General Hospital, Kita 3 Higashi 8, Chuo-ku, Sapporo 060-0033, Japan, Tel: +8111261 5331, E-mail: taka.sato@ja-hokkaidoukouseiren.or.jp

Citation: Sato T (2017) Endoscopic Injection Sclerotherapy for Rectal Varices with Portal Hypertension. Int J Gastroenterol Disord Ther 4: 130. doi: https://doi. org/10.15344/2393-8498/2017/130

Copyright: (c) 2017 Sato. This is an open-access article distributed under the terms of the Creative Commons Attribution License, which permits unrestricted use, distribution, and reproduction in any medium, provided the original author and source are credited. 